\title{
Approximation and Online Algorithms for NFV-Enabled Multicasting in SDNs
}

\author{
Zichuan $\mathrm{Xu}^{\ddagger}$, Weifa Liang ${ }^{\dagger}$, Meitian Huang ${ }^{\dagger}$, Mike Jia ${ }^{\dagger}$, Song Guo ${ }^{\Uparrow}$, and Alex Galis ${ }^{\ddagger}$ \\ $\dagger$ Australian National University, Canberra, ACT 0200, Australia \\ $\ddagger$ University College London, London WC1E 7JE, UK \\ I The Hong Kong Polytechnic University, Hung Hom, Kowloon, Hong Kong SAR \\ Email: z.xu@ucl.ac.uk, wliang@cs.anu.edu.au, u4700480@anu.edu.au,u5515287@anu.edu.au, song.guo@polyu.edu.hk, a.galis@ucl.ac.uk
}

\begin{abstract}
Multicasting is a fundamental functionality of networks for many applications, including online conferencing, event monitoring, video streaming, and system monitoring in data centers. To ensure multicasting reliable, secure and scalable, a service chain consisting of network functions (e.g., firewalls, Intrusion Detection Systems (IDSs), and transcoders) usually is associated with each multicast request, which we refer to as an NFV-enabled multicast request. In this paper we study NFV-enabled multicasting in a Software-Defined Network (SDN) with the aim to minimize the implementation cost of each NFVenabled multicast request and maximize the network throughput of a sequence of NFV-enabled requests, subject to resource capacity constraints on servers and links. We first formulate novel NFV-enabled multicasting and online NFV-enabled multicasting problems. We then devise the very first approximation algorithm with an approximation ratio of $2 K$ for the NFV-enabled multicasting problem if the number of servers for implementing the network functions of each request is no more than a constant $K(\geq 1)$. Furthermore, we also study dynamic admissions of NFV-enabled multicast requests without the knowledge of future request arrivals with the objective to maximize the network throughput, and propose an online algorithm with a competitive ratio of $O(\log n)$ for it if $K=1$, where $n$ is the number of nodes in the network. We finally evaluate the performance of the proposed algorithms through experimental simulations, and experimental results demonstrate that the proposed algorithms outperform other existing heuristics.
\end{abstract}

\section{INTRODUCTION}

Network Function Virtualization (NFV) [2], [3], [7], [16] is emerging as a promising paradigm that is shaping the future networking landscape, by not only bringing the promise of enabling inexpensive and flexible management solutions but also introducing new challenges to the area of network management. Today's data centers and communication networks deploy a variety of intermediary middleboxes, e.g., firewalls, Intrusion Detection Systems (IDSs), proxies, and WAN optimizers, to guarantee the security and performance of data transfers. However, it is very expensive to achieve the benefits of middleboxes in conventional networks, since the middleboxes are typically made by dedicated hardware devices. Underpinned by the NFV technique, Software-Defined Networking (SDN) that separates the control plane from the data plane can be utilized to enable inexpensive and flexible implementation of network functions as software components running in Virtual Machines (VMs), rather than expensive and hard-to-manage hardware middleboxes. Multicasting in SDNs that transmits data from one source to multiple destinations is a fundamental functionality of the networks, which has wide applications, such as video conferencing, multimedia distribution, software updates, and system monitoring in data centers. Such multicast requests usually require to forward their traffic to some middleboxes before reaching their destinations for security and performance concerns. To admit multicast requests with network function requirements to be implemented as VMs, in this paper we study the problem of NFV-enabled multicasting in a software-defined network that is equipped with servers to run the VMs.

Performing NFV-enabled multicasting in an SDN is significantly challenging, as the VMs in servers for network function implementations are located at different places of the SDN, while the locations of servers partially determine the cost of implementing requests. Naive placements of the VMs of each NFV-enabled multicast request at locations that are far away from its source and its destinations may incur a prohibitive communication cost. In addition, multicast requests usually arrive in the network one by one without the knowledge of future request arrivals. This leads to difficulty to estimate dynamic workloads of computing and bandwidth resources at servers and links. The challenges thus are (1) how to jointly find one or multiple servers to implement the network functions and multicast routes for each incoming multicast request while meeting its computing and bandwidth demands, (2) how to design a novel metric that can accurately capture the dynamic resource usage and workload in an SDN, and (3) how to devise an online algorithm to maximize the number of admitted multicast requests, subject to the resource capacity constraints.

Several studies on multicasting in SDNs have been conducted recently [9], [10], [22], [23]. However, among these work, they either do not consider network functions in multicast requests [9], [10], or only dealt with a single multicast request admission [22], [23]. In contrast, we here investigate NFVenabled multicasting, by devising not only an approximation algorithm with a provable approximation ratio for realizing a single NFV-enabled multicast request but also an online algorithm with a guaranteed competitive ratio for the online NFV-enabled multicasting problem.

To the best of our knowledge, we are the first to formulate a novel NFV-enabled multicast problem in SDNs with the aim to minimize its implementation cost, through striving for the fine tradeoff between computing and bandwidth resource consumptions if no more than $K$ servers are employed to implement the service chain of each request. We devise the very first approximation algorithm for the problem. We also study online NFV-enabled multicasting and devise the very first online algorithm with a provable competitive ratio if only one server is deployed for its service chain implementation. The 
key ingredients in the design of both approximation and online algorithms are a series of non-trivial reductions, reducing the problems into other well-known optimization problems.

The main contributions of this paper are as follows.

- We first study the problem of NFV-enabled multicasting in an SDN to minimize the implementation cost of each NFV-enabled multicast request in terms of both computing and bandwidth resource consumptions

- We then devise the very first approximation algorithm with an approximation ratio of $2 K$ for minimizing the implementation cost of the request, assuming that the number of servers used for implementing the service chain of each request is no more than $K$

- We also investigate dynamic admissions of NFVenabled multicast requests without the knowledge of future request arrivals with an aim to maximize the network throughput, by proposing an online algorithm with a provable competitive ratio.

- We finally evaluate the performance of the proposed algorithms through experimental simulations. Experimental results demonstrate that the proposed algorithms outperform other existing heuristics.

The rest of the paper is organized as follows. Section II reviews the related work. Section III introduces the system model, notations, and problem definitions. Section IV devises approximation algorithms for the NFV-enabled multicasting problem with and without capacity constraints. Section V devises an online algorithm for the online NFV-enabled multicasting problem. Section VI evaluates the performance of the proposed algorithms by experimental simulation, and Section VII concludes the paper.

\section{RELATED WORK}

Previous studies have extensively explored the issues on placement and resource allocation for NFVs in SDNs [2], [3], [15], [14], [18], [21]. For example, Moens et al. [18] investigated efficient NFV placements in SDNs, by focusing on a hybrid scenario where some network functions are implemented by dedicated physical hardware and others are implemented in VMs. Lukovszki et al. [15] studied the problem of online admission and embedding of service chains (i.e., a sequence of virtualized network functions) into a substrate network (i.e., an SDN with both bandwidth and computing resource capacities on its links and nodes). Li et al. [14] designed and implemented a system to provide dynamic provisions of resources in an NFV-enabled SDN. They also studied the problem of maximizing the total number of requests that can be assigned to each service chain, by utilizing Integer Linear Programming (ILP) and randomized rounding methods. Cao et al. [1] dealt with policy-aware traffic engineering in SDNs, by assuming that the traffic has to pass a given sequence of network functions. Cohen et al. [3] considered NFV placements in an SDN with and without server capacity constraints for NFV-enabled unicast requests. Kuo et al. [13] studied how to implement a single NFV-enabled unicast request with the end-to-end delay constraint by utilizing existing NFV in servers, and proposed a dynamic programming solution to the problem. These studies however do not consider multicasting in SDNs.

There are studies focusing on multicasting in SDNs [9], [22], [23]. Huang et al. [10] devised the very first online algorithms with provable competitive ratios for online unicasting and multicasting in SDNs, under both node capacities (forwarding table sizes) and link capacities (bandwidth capacities) constraints. However, they do not consider network function requirements of multicast requests. Huang et al. [9] studied the scalability problem for multicasting in SDNs, by proposing an efficient algorithm to find a branch-aware Steiner Tree (BST) for each multicast request. Their solutions however may not be applicable to the NFV-enabled multicasting problem, as the solutions do not consider NFV requirements of requests.

A very closely related work is conducted by Zhang et al. [22], [23]. They investigated the NFV-enabled multicasting problem in SDNs, by assuming that there are sufficient computing and bandwidth resources in the SDN to accommodate any multicast request, for which they provided a 2-approximation algorithm for the problem if only one server $(K=1)$ is deployed for implementing the service chain of each multicast request. However, their method cannot be extended to a general case of the problem, where multiple servers are employed. In reality, both computing and bandwidth resources of an SDN are limited, and need to be carefully allocated. Also, they did not consider the online admissions of multiple multicast requests. Since different requests may have different resource demands, which requests should be admitted/rejected is crucial to optimize the network throughput.

\section{PRELIMINARIES}

In this section, we first introduce the system model, notations and notions, and we then define the problems precisely.

\section{A. System model}

We consider a software-defined network $G=(V, E)$ with a set $V$ of SDN-enabled switch nodes and a set $E$ of links between SDN-enabled switch nodes. Some of the switch nodes in $V$ are attached with computing servers that can implement various network functions as virtual machines (VMs). The communication delay between a switch node and the server attached to it usually is negligible in comparison with the communication delay with other nodes in the network, as they are connected by a high-speed optical fiber. We thus denote by $V_{S}(\subseteq V)$ the subset of switch nodes attached with servers. Notice that each node $v \in V_{S}$ is treated like a switch node without an attached server if its server is not used for implementing VMs. Otherwise, the VM implementation cost of $v$ must be taken into account. Denote by $C_{v}$ and $B_{e}$ the computing capacity of the server attached to a switch node $v \in V_{S}$ and the bandwidth capacity of a link $e \in E$ in $G$, respectively. There is an SDN controller in $G$ that controls the allocations of both the computing and bandwidth resources of $G$ to meet the resource demands of each admitted NFV-enabled multicast request. Fig. 1 is an example of an SDN.

\section{B. NFV-enabled multicast requests and pseudo-multicast trees}

An NFV-enabled multicast request $r_{k}$ is represented by a quadruple $r_{k}=\left(s_{k}, D_{k} ; b_{k}, S C_{k}\right)$, where $s_{k} \in V$ is the source, 


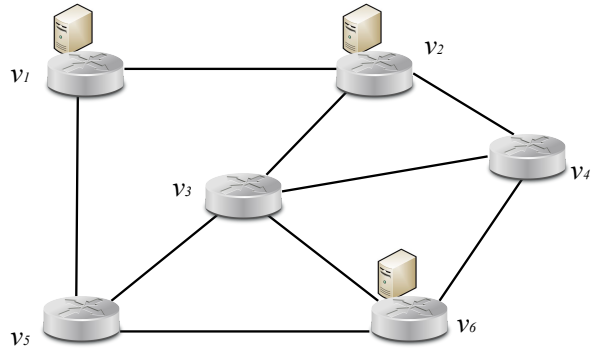

Fig. 1. An SDN $G$ with a set $V=\left\{v_{1}, v_{2}, v_{3}, v_{4}, v_{5}, v_{6}\right\}$ of SDN switches and a subset $V_{S}=\left\{v_{1}, v_{2}, v_{6}\right\}$ of switches with servers.

$D_{k}$ is the set of destinations (or terminals) with $D_{k} \subseteq V, b_{k}$ is the demanded bandwidth by $r_{k}$, and $S C_{k}$ is the service chain of $r_{k}$ that consists of a sequence of network functions that must be implemented by either dedicated hardware middleboxes or virtual machines running on servers. Specifically, the service chain $S C_{k}$ of request $r_{k}$ enforces that every message from the source of $r_{k}$ must go through each of the network functions of the chain in the specified order prior to reaching its destinations, as illustrated in Fig. 2. The network functions in $S C_{k}$ can be implemented by VMs in servers [7], [17], [19]. Without loss of generality, we assume that the network functions in $S C_{k}$ are consolidated to a server in $G$. Specifically, when realizing multicast request $r_{k}$, its message passes a server hosting the VM of its service chain $S C_{k}$, the traffic will be directed to the VM and all the network functions in $S C_{k}$. Denote by $C_{v}\left(S C_{k}\right)$ the amount of demanded computing resource to implement $S C_{k}$ of multicast request $r_{k}$ in server $v \in V_{s}$.

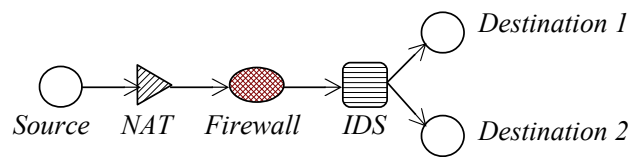

Fig. 2. A service chain $\langle$ NAT, Firewall, IDS $\rangle$.

A pseudo-multicast tree is a graph $G_{T}$ derived from a multicast tree $T$ for the data traffic routing of an NFV-enabled multicast request. We here use an example to illustrated the pseudo-multicast tree concept. Consider a multicast tree $T$ as shown in Fig. 3, where nodes $A$ and $B$ are attached with servers for processing the NFVs in $S C_{k}$ of a multicast request $r_{k}$, the set of destinations of request $r_{k}$ is $\left\{d_{1}, d_{2}, d_{3}, d_{4}, d_{5}\right\}$. Recall that a packet from source $s_{k}$ must pass through a server for processing the NFVs in $S C_{k}$ prior to reaching all destinations. However, in this case, only the destinations $d_{1}$ and $d_{4}$ in $T$ can correctly receive the processed packet, because there are servers at $A$ and $B$ respectively, while the other three destinations $d_{2}, d_{3}$ and $d_{5}$ cannot. To enable the packet to pass through a server before reaching $d_{2}, d_{3}$ and $d_{4}$, the packet routing proceeds as follows. When the packet is processed in node $A$, the processed packet is sent back to node $a$ along the tree path $P_{A, a}$, node $a$ then forwards the processed packet to $d_{2}$ (see Fig. 3(b)). Similarly, the processed packet at $B$ will be sent back to node $b$ along the tree path $P_{B, b}$. Assume that the distance between nodes $A$ and $e$ is greater than the distance between nodes $B$ and $e$, the processed packet at node $b$ will be further forwarded to node $e$. The processed packet will finally reach node $e$ and be forwarded to node $d_{5}$ (see Fig. 3(b)). We term this routing graph derived from $T$ as a pseudo-multicast tree $G_{T}$, as shown by Fig. 3(b). It can be seen that another tree $T^{\prime}$ (see Fig. 3(c)) derived from $G_{T}$ will have the same cost as $G_{T}$, i.e., $c\left(T^{\prime}\right)=c\left(G_{T}\right)$.

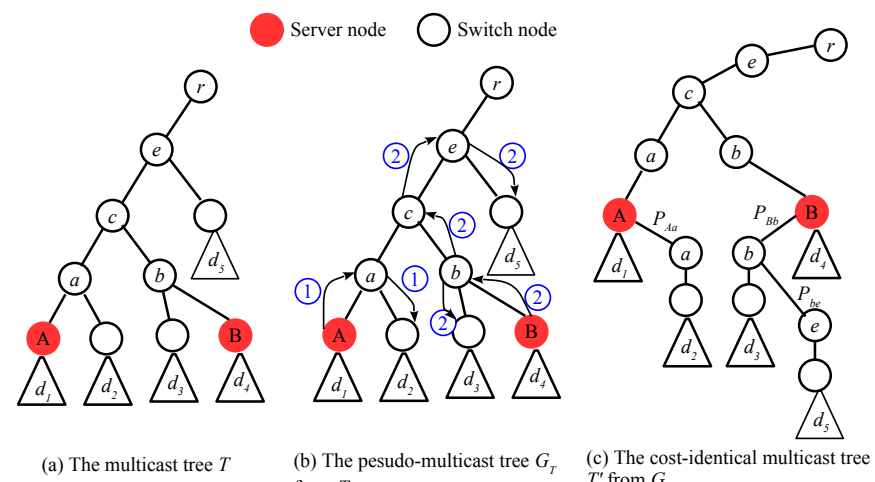
from $T$ $T^{\prime}$ from $G_{T}$

Fig. 3. A pseudo-multicast tree $G_{T}$ derived from a multicast tree $T$ for an NFV-Enabled multicast request $r_{k}$, and another tree $T^{\prime}$ derived from $G_{T}$ is constructed which has the identical cost as $G_{T}$.

\section{Problem definitions}

Given an $\operatorname{SDN} G=(V, E)$ and a multicast request $r_{k}$ $\left(=\left(s_{k}, D_{k} ; b_{k}, S C_{k}\right)\right)$, we consider the following NFV-enabled multicasting problems with and without various resource capacity constraints.

Case 1. The SDN $G=(V, E)$ has sufficient computing and bandwidth resources to meet the resource demands of any NFVenabled multicast request. As the network operator of $G$ then charges each admitted multicast request on a pay-as-you-go basis, its major concern is its operational cost that is defined as the sum of the costs of using computing and bandwidth resources for admitted requests. Let $c_{e}$ and $c_{v}$ be the usage costs of one unit of bandwidth and computing resources at link $e \in E$ and server $v \in V_{S}$, respectively. As the computing resource demand of the service chain of each request usually is within the capacity of each server, we assume that the number of servers, each of which implements an instance of the service chain $S C_{k}$ of each admitted multicast request $r_{k}$, is no more than a constant $K$ with $K \geq 1$. The $N F V$-enabled multicasting problem without SDN resource capacity constraint in $G$ for an NFV-enabled multicast request $r_{k}$ is to find a pseudo-multicast tree such that its implementation cost is minimized, if no more than a constant number $K$ of servers are used for implementing service chain $S C_{k}$, assuming that $G$ has sufficient computing and bandwidth resources in $G$.

Case 2. Both computing and communication resources of $G$ are capacitated. Then, for an incoming NFV-enabled multicast request, the network may or may not have enough resources at that moment to admit it. Or it is too expensive to admit the request, i.e., the request should be rejected. We thus define the NFV-enabled multicasting problem with SDN resource capacity constraint in an $\operatorname{SDN} G=(V, E)$ for an NFV-enabled multicast request $r_{k}$ is to find a pseudo-multicast tree in $G$ for implementing $r_{k}$ such that its implementation cost is minimized, if no more than a constant number $K$ of 
servers will be used for implementing its service chain $S C_{k}$, subject to computing and bandwidth capacity constraints on servers and links of $G$.

Both the defined problems are NP-hard, as their special case - the traditional multicast problem without the NFV constraints is NP-hard [4]. So far we have only considered a single request admission. In reality, the requests arrive into the system one by one without the knowledge of future request arrivals, and we refer to this dynamic request admission as the online request admission. Considering that the limited resources of $G$ may not be able to admit all requests, they need to be dynamically admitted. We thus formulate this dynamic admissions of multicast requests as the online $N F V$-enabled multicasting problem in $G$ to admit as many NFV-enabled multicast requests as possible without the knowledge of future request arrivals, while meeting the computing and bandwidth resource demands of admitted multicast requests, subject to the computing and bandwidth capacity constraints on servers and links of $G$, assuming that no more than $K$ servers are used to implement the service chain of each request.

\section{ApProximation Algorithms FOR THE NFV-enabled Multicasting Problem}

In this section we deal with the NFV-enabled multicasting problem with and without resource capacity constraints.

\section{A. Algorithm overview}

The basic idea of the proposed approximation algorithms is to find a multicast tree rooted at the source and spanning all destinations, and each message from the source to destinations passes through a server in the tree, such that the cost of the tree is minimized. To this end, the finest tradeoff between the computing and communication costs needs to be explored. Specifically, if a server $v$ with a lower computing cost is included in the pseudo-multicast tree for multicast request $r_{k}$, the computing cost of implementing $r_{k}$ may be reduced. This however will increase the communication cost if the chosen server $v$ is far from the destinations of $r_{k}$. Furthermore, if there are multiple servers in different branches of the multicast tree, then the message can pass through each of these servers to reach the destinations in $D_{k}$, and thus leads to less bandwidth usages from the source to the destinations, at the expense of high computing cost. We thus identify a set of servers with each implementing the service chain $S C_{k}$ of $r_{k}$ and find a pseudomulticast tree for the request with the identified server(s) on the path from the source $s_{k}$ to each destination $u \in D_{k}$. As $K$ is a constant, we aim to find a pseudo-multicast tree in $G$ that contains no more than $K$ servers and the path in the tree from $s_{k}$ to each destination $u \in D_{k}$ must pass through one of the identified servers such that the cost of the tree is minimized.

Recall that there are $\left|V_{s}\right|$ switches in $G$ with servers, clearly $K \leq\left|V_{s}\right|$. As a pseudo-multicast tree for any NFV-enabled multicast request can contain at least one but no more than $K$ servers, there are at most $\left(\begin{array}{c}\left|V_{S}\right| \\ K\end{array}\right)$ combinations of servers that can meet the computing resource demand of service chain $S C_{k}$ of request $r_{k}$. For each combination of servers, a pseudo-multicast tree in $G$ can be identified, and the tree with the minimum cost is then used to implement $r_{k}$. Our general strategy is to reduce the multicast tree problem into a Steiner tree problem in an auxiliary undirected graph. An approximate solution to the latter returns an approximate solution to the former.

\section{B. Approximation algorithm without resource capacity con- straints}

Given an NFV-enabled multicast request $r_{k}$, we now devise an approximation algorithm for the NFV-enabled multicasting problem in $G$ without SDN resource capacity constraint, by reducing it to the Steiner tree problem in an auxiliary undirected graph $G_{k}^{i}=\left(V_{k}^{i}, E_{k}^{i} ; c\right)$ with an edge weight function $c$ for all $i$ with $1 \leq i \leq\left(\begin{array}{c}\left|V_{S}\right| \\ K\end{array}\right)$, where $V_{k}^{i}=V \cup\left\{s_{k}^{\prime}\right\}, E_{k}^{i}=$ $E \cup\left\{\left(s_{k}^{\prime}, v\right) \mid v \in V_{S}^{i}\right\}, V_{S}^{i}\left(\subseteq V_{S}\right)$ is the $i$ th combination of the servers in $V_{S}$, and $s_{k}^{\prime}$ is a virtual source of request $r_{k}$. For each $v \in V_{S}^{i}$, if edge $\left(s_{k}, v\right) \in E$ in $G$, the cost of edge $\left(s_{k}, v\right) \in E_{k}^{i}$ is assigned zero. $s_{k}^{\prime}$ now is the new source in $G_{k}^{i}$, replacing the original source $s_{k}$. Notice that the original source $s_{k}$ is still contained in $G_{k}^{i}$ serving as a 'regular' switch node without an attached server. To guarantee that the traffic of $r_{k}$ passes through its service chain $S C_{k}$ that is implemented in one or multiple servers in $V_{S}^{i}\left(\subseteq V_{S}\right)$, we connect $s_{k}^{\prime}$ with all server nodes in $V_{S}^{i}$, where the edge between $s_{k}^{\prime}$ and each server node $v \in V_{S}^{i}$ in $G_{k}^{i}$ represents a shortest path $p_{s_{k}, v}$ in $G$ between nodes $s_{k}$ and $v$. The weight of edge $\left(s_{k}^{\prime}, v\right)$ is the cost sum of the edges in path $p_{s_{k}, v}$ plus the cost of implementing $S C_{k}$ in server $v$, i.e., $c_{\left(s_{k}^{\prime}, v\right)}=\sum_{e \in p_{s_{k}^{\prime}, v}} c_{e} \cdot b_{k}+c_{v}\left(S C_{k}\right)$, where $c_{v}\left(S C_{k}\right)$ is the cost of the amount $C_{v}\left(S C_{k}\right)$ of computing resource consumption for implementing $S C_{k}$. In addition, the weight $c_{e}$ of each edge $e \in E_{k}^{i} \cap E$ is the cost $c_{e} \cdot b_{k}$ of allocating the amount $b_{k}$ of bandwidth resource to request $r_{k}$ on edge $e \in E$. An example of the constructed auxiliary graph $G_{k}^{i}$ that is derived from the SDN in Fig. 1 is shown in Fig. 4.
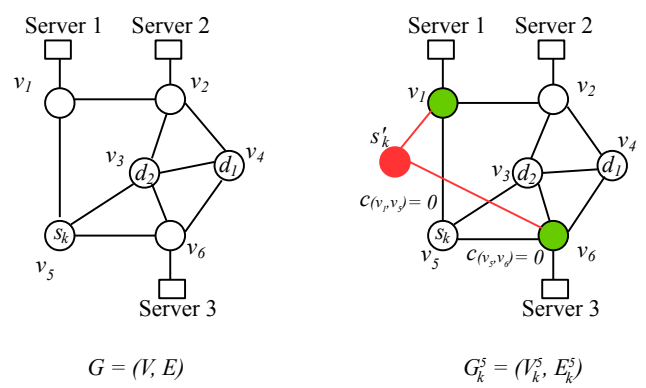

Fig. 4. An example of the auxiliary graph $G_{k}^{5}=\left(V_{k}^{5}, E_{k}^{5}\right)$ constructed from an SDN $G=(V, E)$ with $V_{S}^{5}=\left\{v_{1}, v_{6}\right\}$, assuming that $K=2$ and $V_{S}=\left\{v_{1}, v_{2}, v_{6}\right\}$. There are $\left(\begin{array}{c}\left|V_{S}\right|^{S} \\ K\end{array}\right)=3 \cdot 2=6$ auxiliary graphs derived from $G$, and all different combinations of servers in $V_{S}$ are $V_{S}^{1}=\left\{v_{1}\right\}, V_{S}^{2}=\left\{v_{2}\right\}$, $V_{S}^{3}=\left\{v_{6}\right\}, V_{S}^{4}=\left\{v_{1}, v_{2}\right\}, V_{S}^{5}=\left\{v_{1}, v_{6}\right\}$, and $V_{S}^{6}=\left\{v_{2}, v_{6}\right\}$.

For the sake of convenience, we assume that $V_{S}=$ $\left\{v_{1}, v_{2}, \ldots, v_{\left|V_{S}\right|}\right\}$. Having constructed auxiliary graph $G_{k}^{i}$, we find a Steiner tree in $G_{k}^{i}$ for request $r_{k}$. Specifically, we first find a minimum spanning tree $T_{m s t}^{i}$ in a complete graph consisting of nodes in $\left\{s_{k}^{\prime}\right\} \cup D_{k}$, in which each edge is assigned a weight that is equal to the length of the shortest path in $G_{k}^{i}$ between its two endpoints. Let $H_{k}^{i}$ be a subgraph of $G_{k}^{i}$ derived from $T_{m s t}^{i}$ by replacing each edge of $T_{m s t}^{i}$ with its corresponding shortest path in $G_{k}^{i}$. We then find an approximate Steiner tree $T_{k}^{i}$ in $H_{k}^{i}$ by applying the approximation algorithm due to Kou et al. [12], which will serve as the multicast tree for $r_{k}$. The 
detailed description of the algorithm is given in Algorithm 1.

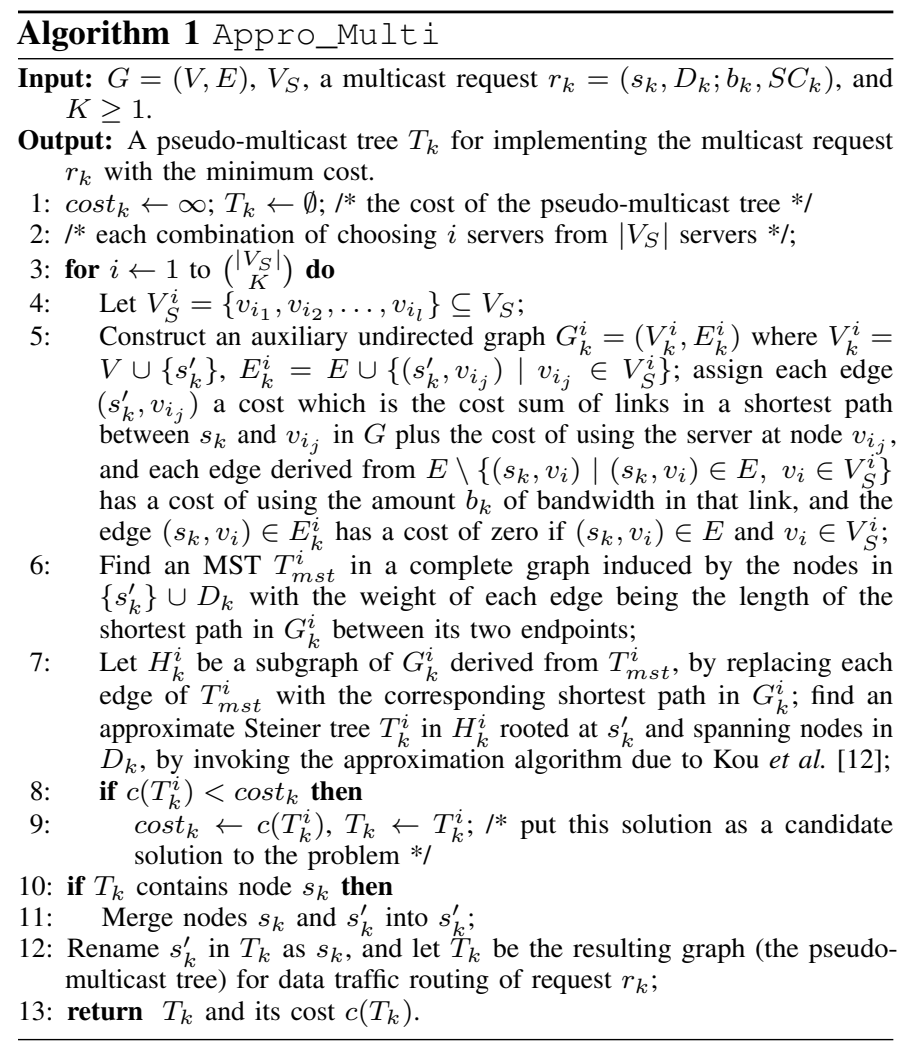

\section{Approximation algorithm with resource capacity constraints}

We now deal with the NFV-enabled multicasting problem under computing and bandwidth resource capacity constraints, by performing some minor modifications to Algorithm 1 . Specifically, a subgraph $G^{\prime}=\left(V^{\prime}, E^{\prime}\right)$ of $G$ is constructed, where $V^{\prime}=V, E^{\prime}=\{(u, v) \mid(u, v) \in E$, and the residual bandwidth at link $(u, v)$ is no less than $\left.b_{k}\right\}$, a subset set $V_{S}^{\prime}$ of $V_{S}$ will be used, and $V_{S}^{\prime}=\left\{v_{i} \mid v_{i} \in V_{S}\right.$ if the available computing resource at $v_{i}$ can meet the computing resource demands of $\left.r_{k}\right\}$. Algorithm 1 then is applied to graph $G^{\prime}$, using the server set $V_{S}^{\prime}$. Clearly, all the resource demands by $r_{k}$ will be met. In case $G^{\prime}$ is disconnected, and none of its connected components contains the source node and all destinations of $r_{k}$ and at least one server node, then the request should be rejected, because there are no sufficient resources in $G$ for its implementation. For simplicity, this algorithm is referred to as algorithm Appro_Multi_Cap.

\section{Algorithm analysis}

We now show the correctness of Algorithm 1, and analyze its time complexity and the approximation ratio. The analysis under resource capacity constraints can be similarly performed, and thus omitted.

Theorem 1: Given an $\operatorname{SDN} G=(V, E)$, a set $V_{S}$ of switch nodes with each having an attached server, and an NFVenabled multicast request $r_{k}=\left(s_{k}, D_{k} ; b_{k}, S C_{k}\right)$, there is an approximation algorithm, Algorithm 1, for the NFV-enabled multicasting problem with and without SDN resource capacity constraints, which delivers an approximate solution with an approximation ratio of $2 K$, assuming no more than $K$ servers will be employed for its service chain implementation. The time complexity of the algorithm is $O\left(|V|^{3} \cdot\left|V_{S}\right|^{K}\right)$, where $\left|V_{S}\right| \ll|V|$ and $K \geq 1$ is a small integer.

Proof: Clearly, according to the construction of $G_{k}^{i}$, the solution delivered by Algorithm 1 is feasible.Due to space limitation, the detailed proof for feasibility is omitted.

We now analyze the approximation ratio of Algorithm 1 . Let $G_{T}^{*}$ be the optimal pseudo-multicast tree for the NFVenabled multicast request $r_{k}$ in $G$. If $G_{T}^{*}$ is not a multicast tree, there is a corresponding tree $T^{\prime}$ with the identical cost as $G_{T}^{*}$, following the transformation in Section III; otherwise $G_{T}$ itself is a multicast tree. From now on, we denote by $T^{*}$ either the optimal multicast tree $G_{T}^{*}$ or its corresponding costidentical tree $T^{\prime}$. We assume that there are $l$ servers in $T^{*}$ for implementing $S C_{k}$ with $1 \leq l \leq K$. Without loss of generality, we assume that these $l$ nodes are $v_{1}, v_{2}, \ldots, v_{l}$, respectively. Clearly, it can be easily shown that none of pairs of these nodes in $T^{*}$ has the ancestor and descendant relationship in terms of a node being used as a server, otherwise the node in $V_{S}$ will be treated as a regular switch node without the use of its server. Each subtree $T_{v_{i}}^{*}$ of $T^{*}$ rooted at $v_{i}$ contains some destinations, and all of the $l$ subtrees will contain all the destinations in $D_{k}$, following its definition. We construct another tree $T_{c}^{*}=\left(V^{\prime}, E^{\prime}\right)$ which is derived from $T^{*}$ by compressing the path in $T^{*}$ from $s_{k}$ to each node $v_{i}$ as follows. We replace the source node $s_{k}$ by a node $s_{k}^{\prime}$ and the path in $T^{*}$ from $s_{k}$ to $v_{i}$ by an edge $\left(s_{k}^{\prime}, v_{i}\right)$, and assign the edge a weight that is the sum of all edge costs in the path plus the cost of using server $v_{i}$. We now claim that the cost of tree $T_{c}^{*}$ is no greater than $l$ times the cost of tree $T^{*}$, i.e., $c\left(T_{c}^{*}\right) \leq l \cdot c\left(T^{*}\right)$ by showing the claim as follows.

It can be seen that there is a multicast tree $T_{k}^{i}$ in $G_{k}^{i}$ rooted at source $s_{k}^{\prime}$ and spanning all destinations in $D_{k}$, which has the same topological structure as $T_{c}^{*}$, however, it has a lower cost compared with that of $T_{c}^{*}$, i.e., $c\left(T_{k}^{i}\right) \leq c\left(T_{c}^{*}\right)$. This is because the weight of each edge in $G_{k}^{i}$ between $s_{k}^{\prime}$ and $v_{i}$ is the length of the shortest path in $G$ between the two nodes plus the cost of using server $v_{i}$, while the corresponding edge weight in $T_{c}^{*}$ is the sum of all edge weights in the path in $T^{*}$ between $s_{k}$ and $v_{i}$ plus the cost of using server $v_{i}$.

Let $T_{k}^{O P T, i}$ be an optimal multicast tree in $G_{k}^{i}$ rooted at $s_{k}^{\prime}$ and spanning all destinations in $D_{k}$ and each path in the tree from $s_{k}^{\prime}$ to a destination goes through one of the servers in $V_{S}^{\prime}$. Then, $c\left(T_{k}^{O P T, i}\right) \leq c\left(T_{k}^{i}\right)$ as $T_{k}^{i}$ is one of the multicast trees for multicast request $r_{k}$. Let $T_{k}^{a p p, i}$ be an approximate multicast tree in $G_{k}^{i}$ for multicast request $r_{k}$ by the algorithm due to Kou et al. [12], then $c\left(T_{k}^{a p p, i}\right) \leq 2 c\left(T_{k}^{O P T, i}\right) \leq 2 c\left(T_{k}^{i}\right) \leq$ $2 c\left(T_{c}^{*}\right)=2 \cdot l \cdot c\left(T^{*}\right)$. Since a pseudo-multicast tree $T_{k}$ with the minimum cost from the $\left(\begin{array}{c}\left|V_{S}\right| \\ K\end{array}\right)$ auxiliary undirected graphs $G_{k}^{i}$ for all $i$ with $1 \leq i \leq\left(\begin{array}{c}\left|V_{S}\right| \\ K\end{array}\right)$ will be found and the value of $l$ is within $[1, K]$, the cost of the pseudo-multicast tree $T_{k}$ for $r_{k}$ is no greater than $2 K \cdot c\left(T^{*}\right)$.

The analysis of the time complexity of Algorithm 1 is omitted, due to space limitation. 


\section{ONLINE AlgorithM FOR THE ONLINE NFV-ENABLED MULTICASTING PROBLEM}

We now study the online NFV-enabled multicasting problem in $G$ with SDN resource capacity constraints. We first propose a novel cost model to capture dynamic resource consumptions in $G$. We then devise an online algorithm with a competitive ratio for the problem when $K=1$.

\section{A. Cost model}

Given an $\operatorname{SDN} G=(V, E)$ with limited computing and bandwidth capacities at its servers and links, there is a need of a metric to capture dynamic consumptions of its resources in order to better utilize the resources, by encouraging the use of underloaded resources while restricting the use of overloaded resources to maximize the number of NFV-enabled multicast request admissions. A simple cost model which is referred to the linear cost model, is widely adopted to charge each request with a cost that is linearly proportional to the amount of its resource consumption, regardless of whether a specific resource has been overloaded or underloaded. Clearly, this model may lead to some resources being under-utilized while others being over-utilized. Consequently, significant number of requests may be rejected due to unbalanced resource utilizations.

We here introduce a novel cost model that assigns an underloaded resource with a lower cost and an overloaded resource with a higher cost. Thus, the resources in the network can be maximally allocated among user requests, thereby maximizing the network throughput. Specifically, let $C_{v}(k)$ be the amount of available computing resource at the server attached to a switch node $v \in V_{S}$ and $B_{e}(k)$ the amount of available bandwidth at link $e \in E$ respectively, when multicast request $r_{k}$ arrives. To capture the resource use of $r_{k}$, we use exponential functions to represent the costs $c_{v}(k)$ and $c_{e}(k)$ of its usages of computing and bandwidth resources at server node $v$ and link $e$ :

$$
c_{v}(k)=C_{v}\left(\alpha^{1-\frac{C_{v}(k)}{C_{v}}}-1\right),
$$

where $\alpha$ is a constant with $\alpha>1, C_{v}(k)=C_{v}(k-1)-$ $C_{v}\left(S C_{k}\right)$ if $r_{k}$ is admitted, and $C_{v}(0)=C_{v}$ initially. (1$\left.\frac{C_{v}(k)}{C_{v}}\right)$ in Eq. (1) is the utilization ratio of computing resource at server $v \in V_{S}$. The rationale behind is that the use of less residual computing resource will be charged with a higher cost, while the use of plenty of residual computing resource will be charged with a much less cost.

The cost $c_{e}(k)$ of using the bandwidth resource at link $e$ prior to the admission of $r_{k}$ can be similarly defined, i.e.,

$$
c_{e}(k)=B_{e}\left(\beta^{1-\frac{B_{e}(k)}{B_{e}}}-1\right),
$$

where $\beta$ is a constant with $\beta>1, B_{e}(k)=B_{e}(k-1)-b_{k}$ if $r_{k}$ is admitted, and $B_{e}(0)=B_{e}$ initially. We will use the defined costs to guide the network resource allocations to meet the demands of NFV-enabled multicast requests.

\section{B. Online algorithm}

We now propose an online algorithm with a provable competitive ratio for the online NFV-enabled multicasting problem, assuming the service chain of each multicast request is implemented by a single server, i.e., $K=1$.
The basic idea behind the algorithm is to determine whether every incoming NFV-enabled multicast request $r_{k}$ will be admitted or rejected, depending on a given admission control policy. If $r_{k}$ is admissible, the algorithm requires to jointly find a server with sufficient computing resource to implement the service chain $S C_{k}$ and a pseudo-multicast tree for $r_{k}$, such that the cost of implementing the request is minimized, subject to the resource capacity constraints on the network. For the finding of such a pseudo-multicast tree, we have an important observation: one of the $\left|V_{S}\right|$ servers must be contained in any pseudo-multicast tree for each request $r_{k}$, the pseudo-multicast tree must include the server as one of the destination nodes of $r_{k}$. Thus, we can find a Steiner tree in $G_{k}=\left(V_{k}, E_{k} ; w\right)$ for request $r_{k}$ with source $s_{k}$ and the destination set $D_{k} \cup\{v\}$, where $v \in V_{S}$ has sufficient computing resource. Notice that $G_{k}\left(V_{k}, E_{k} ; w\right)$ is an undirected graph that is identical to $G(V, E)$, i.e., $V_{k}=V$ and $E_{k}=E$. The weight $w_{e}(k)$ of each edge $e \in E_{k}$ is the normalized cost of the cost defined by Eq. (2), that is, $w_{e}(k)=c_{e}(k) / B_{e}$, while the weight $w_{v}(k)$ of each node $v \in V_{S}$ for $r_{k}$ is the normalized cost of the cost defined by Eq. (1), i.e, $w_{e}(k)=c_{v}(k) / C_{v}$. Let $T$ be an approximate Steiner tree in $G_{k}$ rooted at $s_{k}$ and spanning the terminals in $D_{k}$ by the approximation algorithm due to Kou et al. [12], where $G_{k}$ is the graph $G(V, E)$ that considered the first $k-1$ requests already, partial resources at its servers and links are occupied by some of the first $(k-1)$ requests at this moment. We build a multicast tree for $r_{k}$. If server $v$ is in any path in $T$ from $s_{k}$ to each destination, $T$ is the multicast tree, and its cost is no more than twice the optimal one [12]; otherwise, assume that the path between $s_{k}$ and a destination node $d$ does not contain server $v$. Let $u$ be the lowest common ancestor $u=L C A(v, d)$ between nodes $v$ and $d$ in $T$. Then, when the message from $s_{k}$ is sent to server $v$ for processing, the processed message continues forwarding to all destinations in the subtree rooted at $v$; for the destination $d \in D_{k}$, the processed message at node $v$ is then sent back to node $u$, which then forwards toward destination $d$. Clearly, in the worst scenario, the processed message will be sent back to the source $s_{k}$ for multicasting.

Let $T$ and $T^{*}$ be the found approximate Steiner tree by the approximation algorithm in [12] and the optimal one. Denote by $T_{k}$ and $T_{k}^{*}$ the pseudo-multicast tree based on $T$ and the optimal multicast tree, respectively. The sum of the weights of the pseudo-multicast tree $T_{k}$ based on $T$ and server $v$ is

$$
\begin{aligned}
& w\left(T_{k}\right)+w_{v}(k)=w(T)+w\left(P_{v, u}^{T}\right)+w_{v}(k) \leq w(T) \\
& +w\left(P_{v, s_{k}}^{T}\right)+w_{v}(k) \leq 2 w(T)+w_{v}(k) \leq 2\left(w(T)+w_{v}(k)\right) \\
& \leq 4 w\left(T^{*}\right)+2 w_{v}(k) \leq 4\left(w\left(T^{*}\right)+w_{v}(k)\right) \leq 4 O P T_{v},
\end{aligned}
$$

where $P_{x, y}^{T}$ is a path in $T$ between node $x$ and node $y$, and $O P T_{v}$ is the optimal cost of the pseudo-multicast tree using server $v$ as its service chain processing server. Thus, the optimal solution $O P T$ for request $r_{k}$ in $G_{k}$ thus is $O P T=\min _{v \in V_{S}}\left\{O P T_{v}\right\}$.

We then adopt the following admission control policy to guide the admission of each multicast request $r_{k}$ : (a) If $w_{v}(k) \geq \sigma_{v}$ for any $v \in V_{S} \cap T_{k}, r_{k}$ will be rejected; and (b) if $\sum_{e \in T_{k}} w_{e}(k) \geq \sigma_{e}, r_{k}$ will be rejected, where $T_{k}$ is a pseudo-multicast tree delivered by an algorithm in $G_{k}$ for $r_{k}, \sigma_{v}>0$ and $\sigma_{e}>0$ are admission control thresholds of computing and bandwidth respectively, and $\sigma_{v}=\sigma_{e}=|V|-1$. 
The detailed online algorithm, referred to as Online_CP, is given in Algorithm 2.

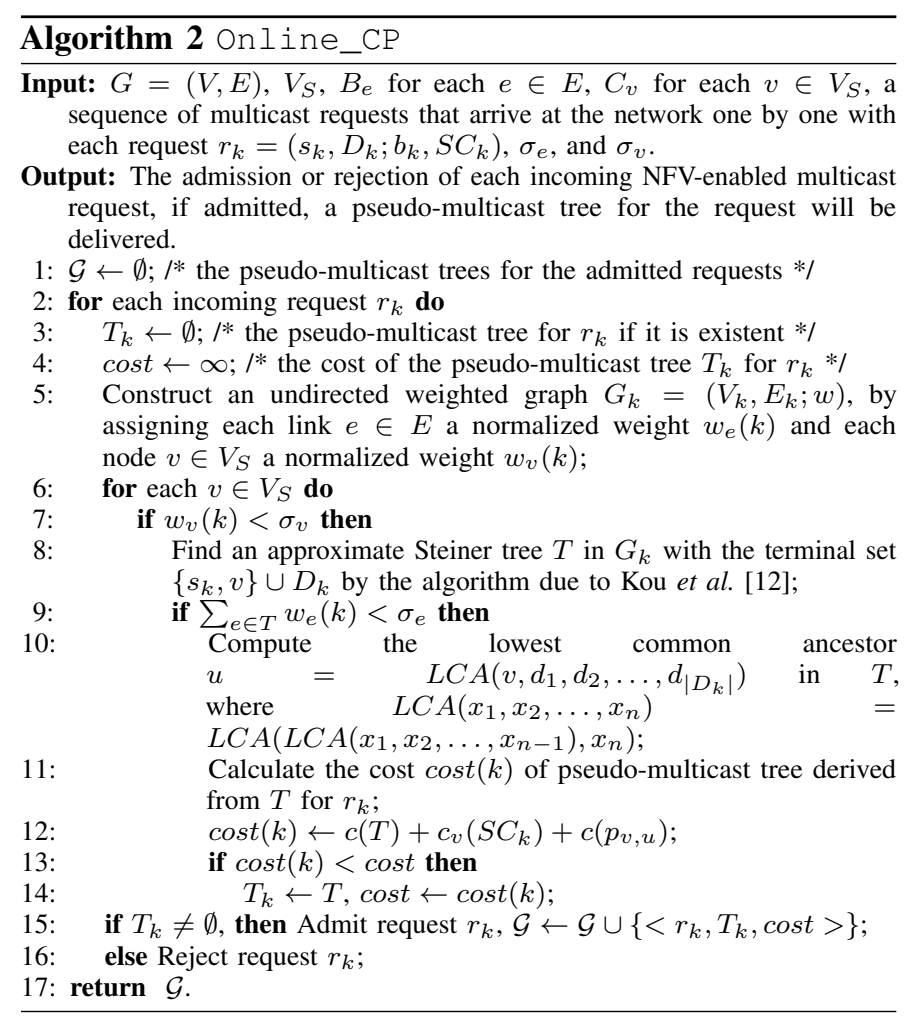

\section{Algorithm analysis}

We now analyze the competitive ratio of algorithm Online_CP. Let $\mathcal{S}(k)$ and $O P T$ be the sets of admitted multicast requests by algorithm Online_CP and an optimal offline algorithm when multicast request $r_{k}$ arrives. Let $\mathcal{R}(k)$ be the set of multicast requests that are rejected by algorithm Online_CP while admitted by the optimal offline algorithm. Then the competitive ratio of algorithm Online_CP is $\frac{|\mathcal{S}(k)|}{|\mathcal{S}(k) \cap O P T|+|\mathcal{R}(k)|} \geq \frac{|\mathcal{S}(k)|}{|\mathcal{R}(k)|+|\mathcal{S}(k)|}$, since $O P T=\mathcal{R}(k) \cup(O P T \cap \mathcal{S}(k)) \subseteq \mathcal{R}(k) \cup S(k)$. Specifically, the analysis of the competitive ratio of algorithm Online_CP consists of three steps: (1) show the upper bound on the accumulative computing and bandwidth resources occupied by requests in $\mathcal{S}(k)$; (2) show the lower bound on the accumulative computing and bandwidth resources occupied by requests in $\mathcal{R}(k)$ ) and (3) derive the competitive ratio by combining the results of steps (1) and (2). We then have the following lemma.

Lemma 1: When multicast request $r_{k}$ arrives, the cost sums of all servers in $V_{S}$ and all links in $E$ are $\sum_{v \in V_{S}} c_{v}(k) \leq$ $2 \mathbb{C}(k) \cdot \log \alpha \cdot(|V|-1)$, and $\sum_{e \in E} c_{e}(k) \leq 2 \mathbb{B}(k) \cdot \log \beta \cdot(|V|-$ $1)$, provided that $b_{k^{\prime}} \leq \frac{\min _{e \in E} B_{e}}{\log \beta}$ and $C_{v}\left(S C_{k^{\prime}}\right) \leq \frac{\min _{v \in V_{S}} C_{v}}{\log \alpha}$ with $1 \leq k^{\prime} \leq k$, where $\mathbb{B}(k)$ and $\mathbb{C}(k)$ are the accumulative amounts of bandwidth and computing resources being occupied by the admitted requests in $\mathcal{S}(k)$, respectively.

Proof: Consider any $k^{\prime} \in \mathcal{S}(k)$ admitted by the online algorithm, its data traffic is first routed to a server $v \in V_{S}$ that hosts its service chain $S C_{k}$ via path $p_{s_{k}, v}$ in $G$, and then to each of its destinations in $D_{k}$ through a subtree $T_{v}$ with root $v$. The costs of computing resource usage of different servers in $V_{S}$ are different. We thus have

$$
\begin{aligned}
& c_{v}\left(k^{\prime}+1\right)-c_{v}\left(k^{\prime}\right) \leq C_{v}\left(\alpha^{1-\frac{C_{v}\left(k^{\prime}+1\right)}{C_{v}}}-\alpha^{1-\frac{C_{v}\left(k^{\prime}\right)}{C_{v}}}\right) \\
& =C_{v} \alpha^{1-\frac{C_{v}\left(k^{\prime}\right)}{C_{v}}}\left(\alpha^{\frac{C_{v}\left(S C_{k^{\prime}}\right)}{C_{v}}}-1\right) \\
& =C_{v} \alpha^{1-\frac{C_{v}\left(k^{\prime}\right)}{C_{v}}}\left(2^{\frac{C_{v}\left(S C_{k^{\prime}}\right) \log \alpha}{C_{v}}}-1\right) \\
& \leq C_{v} \alpha^{1-\frac{C_{v}\left(k^{\prime}\right)}{C_{v}}}\left(\frac{C_{v}\left(S C_{k^{\prime}}\right) \log \alpha}{C_{v}}\right) \\
& =\alpha^{1-\frac{C_{v}\left(k^{\prime}\right)}{C_{v}}} C_{v}\left(S C_{k^{\prime}}\right) \cdot \log \alpha .
\end{aligned}
$$

Notice that the derivation from Eq. (4) to inequality (5) is due to that $2^{x}-1 \leq x$ for $0 \leq x \leq 1$. Similarly, if edge $e \in E$ is in the route for multicast request $r_{k}$, we have

$$
c_{e}\left(k^{\prime}+1\right)-c_{e}\left(k^{\prime}\right) \leq \beta^{1-\frac{B_{e}\left(k^{\prime}\right)}{B_{e}}} b_{k^{\prime}} \cdot \log \beta .
$$

Notice that $c_{v}(0)=0$ for all $v \in V_{S}$. If an NFV-enabled multicast request $r_{k^{\prime}}$ is admitted, this means that

$$
w_{v}\left(k^{\prime}\right)=\alpha^{1-\frac{C_{v}\left(k^{\prime}\right)}{C_{v}}}-1 \leq \sigma_{v}=|V|-1,
$$

We now calculate the cost sum of all edges and the server attached to $v \in V_{S}$ when admitting the multicast request $r_{k}$. Notice that if an edge in $E$ is not included in $T_{k}$ for $r_{k}$, its cost does not change after the admission of $r_{k}$. So does the cost of a server that is not used for implementing $S C_{k}$ of $r_{k}$. Assuming that $v$ is chosen for $r_{k^{\prime}}$, we have

$$
\begin{aligned}
& \sum_{v \in V_{S}} c_{v}\left(k^{\prime}+1\right)-c_{v}\left(k^{\prime}\right)=c_{v}\left(k^{\prime}+1\right)-c_{v}\left(k^{\prime}\right) \\
& \leq\left(\alpha^{1-\frac{C_{v}\left(k^{\prime}\right)}{C_{v}}}\right) C_{v}\left(S C_{k^{\prime}}\right) \log \alpha, \quad \text { by inequality (6) } \\
& =\left(\alpha^{1-\frac{C_{v}\left(k^{\prime}\right)}{C_{v}}}-1\right) C_{v}\left(S C_{k^{\prime}}\right) \log \alpha+C_{v}\left(S C_{k^{\prime}}\right) \log \alpha \\
& \leq\left(\alpha^{1-\frac{C_{v}\left(k^{\prime}\right)}{C_{v}}}-1\right) C_{v}\left(S C_{k^{\prime}}\right) \log \alpha+(|V|-1) C_{v}\left(S C_{k^{\prime}}\right) \log \alpha \\
& \leq 2 C_{v}\left(S C_{k^{\prime}}\right) \log \alpha(|V|-1) \quad \text { by ince }|V|>1
\end{aligned}
$$

Similarly we have

$$
\sum_{e \in E} c_{e}\left(k^{\prime}+1\right)-c_{e}\left(k^{\prime}\right) \leq 2 b_{k^{\prime}} \log \beta(|V|-1) .
$$

We finally have

$$
\begin{aligned}
& \sum_{v \in V_{S}} c_{v}(k)=\sum_{k^{\prime}=1}^{k-1} \sum_{v \in V_{S}}\left(c_{v}\left(k^{\prime}+1\right)-c_{v}\left(k^{\prime}\right)\right) \\
& \leq \sum_{k^{\prime} \in \mathcal{S}(k)} 2 C_{v}\left(S C_{k^{\prime}}\right) \cdot \log \alpha \cdot(|V|-1)=2 \mathbb{C}(k) \cdot \log \alpha \cdot(|V|-1),
\end{aligned}
$$

and similarly $\sum_{e \in E} c_{e}(k) \leq 2 \mathbb{B}(k) \cdot \log \beta \cdot(|V|-1)$.

We now show the lower bound on the cost of a multicast request in $\mathcal{R}(k)$ that is rejected by online algorithm Online_CP but admitted by an optimal offline algorithm.

Lemma 2: For $r_{k^{\prime}} \in \mathcal{R}(k)$, if $\alpha=\beta=2|V|$, we have

$$
w\left(T_{k^{\prime}}^{\prime}\right)+w_{v^{\prime}}\left(k^{\prime}\right) \geq \frac{|V|-1}{4},
$$


where $T_{k^{\prime}}^{\prime}$ is the Steiner tree found by the optimal offline algorithm to route the traffic of $r_{k^{\prime}}$, and $v^{\prime}$ is the switch in $T_{k^{\prime}}^{\prime}$ whose server is selected to implement server chain $S C_{k^{\prime}}$, assuming that both the following inequalities are met: $b_{k^{\prime}} \leq$ $\frac{\min _{e \in E} B_{e}}{\log \beta}$, and $C_{v}\left(S C_{k^{\prime}}\right) \leq \frac{\min _{v \in V_{S}} C_{v}}{\log \alpha}$ with $1 \leq k^{\prime} \leq k$.

Proof: A multicast request $r_{k^{\prime}}$ will be rejected by the proposed online algorithm, Algorithm Online_CP, because of the following cases. Case 1, there is no sufficient computing resource for implementing the service chain of $r_{k^{\prime}}$; Case 2 . There is no sufficient bandwidth resource for routing the traffic of $r_{k^{\prime}}$ to its destinations; Case 3. The weighted sum of edges in the pseudo-multicast tree for $r_{k^{\prime}}$ is too high (Step 9), and/or the weight of the selected server attached to switch $v$ to implement the service chain of $r_{k^{\prime}}$ is too high (Step 7).

Case 1. Let $v^{\prime}$ be the switch whose attached server is selected to implement the service chain of request $k^{\prime}$. As the request is rejected by algorithm Online_CP due to insufficient available computing resource, we have $C_{v^{\prime}}\left(k^{\prime}\right)<C_{v}\left(k^{\prime}\right)$. Therefore, $1-\frac{C_{v^{\prime}}\left(k^{\prime}\right)}{C_{v^{\prime}}} \geq 1-\frac{C_{v}\left(k^{\prime}\right)}{C_{v}\left(S C_{k^{\prime}}\right)} \geq 1-\frac{1}{\log \alpha}$, since $C_{v}\left(S C_{k}\right) \leq \frac{\min _{v \in V_{S}} C_{v}}{\log \alpha}$. We then have

$$
\begin{gathered}
w\left(T_{k^{\prime}}^{\prime}\right)+w_{v^{\prime}}\left(k^{\prime}\right) \geq \alpha^{1-\frac{C_{v^{\prime}}\left(k^{\prime}\right)}{{ }^{C}{ }_{v^{\prime}}}}-1>\alpha^{1-\frac{1}{\log \alpha}}-1 \\
=\frac{\alpha}{2}-1=|V|-1 .
\end{gathered}
$$

Case 2. We have $w\left(T_{k^{\prime}}^{\prime}\right)+w_{v^{\prime}}\left(k^{\prime}\right) \geq \sum_{e \in T_{k^{\prime}}^{\prime}} \beta^{1-\frac{B_{e}\left(k^{\prime}\right)}{B_{e}}}-$ $1 \geq|V|-1$. The derivation is similar to Case 1 . Due to space limitation, omitted.

Case 3. Let $T_{k^{\prime}}$ be the pseudo-multicast tree by algorithm Online_CP for multicast request $r_{k^{\prime}}$. According to inequality (3), we have $w\left(T_{k^{\prime}}\right)+w_{v^{\prime}}\left(k^{\prime}\right) \leq 4\left(w\left(T_{k^{\prime}}^{*}\right)+\right.$ $\left.w_{v^{\prime}}\left(k^{\prime}\right)\right) \leq 4\left(w\left(T_{k^{\prime}}^{\prime}\right)+w_{v^{\prime}}\left(k^{\prime}\right)\right)$, where $T_{k^{\prime}}^{*}$ and $v^{\prime}$ are the optimal multicast tree and the server for $r_{k^{\prime}}$ by the optimal solution. Since the proposed online algorithm rejected the request, we have $w\left(T_{k^{\prime}}\right)>\sigma_{e}$ and/or $w_{v^{\prime}}\left(k^{\prime}\right)>\sigma_{v}$. If $w\left(T_{k^{\prime}}\right)>\sigma_{e}$, we have $w\left(T_{k^{\prime}}^{\prime}\right)+w_{v^{\prime}}\left(k^{\prime}\right) \geq \frac{w\left(T_{k^{\prime}}\right)+w_{v^{\prime}}\left(k^{\prime}\right)}{4} \geq$ $\frac{w\left(T_{k^{\prime}}\right)}{4} \geq \frac{\sigma_{e}}{4}=\frac{|V|-1}{4}$, similarly, if $w_{v^{\prime}}\left(k^{\prime}\right)>\sigma_{v}$, we have $w\left(T_{k^{\prime}}^{\prime}\right)+w_{v^{\prime}}\left(k^{\prime}\right) \geq \frac{\sigma_{v}}{4}=\frac{|V|-1}{4}$. Summarizing the three cases, we have $w\left(T_{k^{\prime}}^{\prime}\right)+w_{v^{\prime}}\left(k^{\prime}\right) \geq \frac{|V|-1}{4}$.

We finally analyze the competitive ratio of Online_CP.

Theorem 2: Given an SDN $G=(V, E)$ with computing capacity $C_{v}$ of each server node $v \in V_{S}$ and bandwidth capacity $B_{e}$ for each link $e \in E$, a sequence of NFV-enabled multicast requests with the $k$ th multicast request $r_{k}$ being represented by a quadruple $\left(s_{k}, D_{k} ; b_{k}, S C_{k}\right)$, there is an online algorithm, Algorithm Online_CP, with a competitive ratio of $O(\log |V|)$ for the online NFV-enabled multicasting problem if only one server is contained in the pseudo-multicast tree for the service chain implementation of the request, provided that $b_{k^{\prime}} \leq \frac{\min _{e \in E} B_{e}}{\log \beta}$ and $C_{v}\left(S C_{k^{\prime}}\right) \leq \frac{\min _{v \in V_{S}} C_{v}}{\log \alpha}$ with $1 \leq k^{\prime} \leq k$. The algorithm takes $O\left(k|V|^{3}\right)$ time if the request sequence contains $k$ NFV-enabled multicast requests.

Proof: The competitive ratio of algorithm Online_CP is analyzed as follows. Let $T_{k^{\prime}}^{*}$ be the optimal multicast tree by the optimal offline algorithm for request $r_{k^{\prime}} \in \mathcal{R}(k)$ and $v^{*} \in T_{k^{\prime}}^{*}$ be the server for the service chain $S C_{k^{\prime}}$ of $r_{k^{\prime}}$.

$$
\begin{aligned}
& \frac{|V|-1}{4}(|\mathcal{R}(k)|) \leq \sum_{r_{k^{\prime}} \in \mathcal{R}(k)} \frac{|V|-1}{4} \\
& \leq \sum_{r_{k^{\prime}} \in \mathcal{R}(k)} \sum_{e \in T_{k^{\prime}}^{*}}\left(\beta^{1-\frac{B_{e}\left(k^{\prime}\right)}{B_{e}}}-1\right)+\alpha^{1-\frac{C_{v^{*}\left(k^{\prime}\right)}}{C_{v^{*}}}}-1, \text { by Lemma } 2 \\
& =\sum_{r_{k^{\prime}} \in \mathcal{R}(k)}\left(\sum_{e \in T_{k^{\prime}}^{*}} c_{e}\left(k^{\prime}\right) / B_{e}+c_{v^{*}}\left(k^{\prime}\right) / C_{v^{*}}\right), \\
& \leq \sum_{r_{k^{\prime}} \in \mathcal{R}(k)}\left(\sum_{e \in T_{k^{\prime}}^{*}} c_{e}(k) / B_{e}+c_{v^{*}}(k) / C_{v^{*}}\right), \\
& \leq \sum_{e \in T_{k^{\prime}}^{*}} c_{e}(k) \sum_{r_{k^{\prime}} \in \mathcal{R}(k)} 1 / B_{e}+c_{v^{*}}(k) \sum_{r_{k^{\prime}} \in \mathcal{R}(k)} 1 / C_{v^{*}} \\
& \leq \sum_{e \in E} c_{e}(k)+\sum_{v \in V_{S}} c_{v}(k) .
\end{aligned}
$$

Following inequalities (13) and Lemma (1), we have

$$
\begin{aligned}
& \frac{|V|-1}{4}(|\mathcal{R}(k)|)<\sum_{e \in E} c_{e}(k)+\sum_{v \in V_{S}} c_{v}(k) \\
& \leq 2 \mathbb{C}(k) \log \alpha(|V|-1)+2 \mathbb{B}(k) \log \beta(|V|-1) \\
& \leq 2|\mathcal{S}(k)| C_{\max } \log \alpha(|V|-1)+2|\mathcal{S}(k)| b_{\max } \log \beta(|V|-1) \\
& =2|\mathcal{S}(k)|(|V|-1)\left(C_{\text {max }} \log \alpha+b_{\text {max }} \log \beta\right),
\end{aligned}
$$

where $C_{\max }=\arg \max _{k} C_{v}\left(S C_{k}\right)$ and $b_{\max }=\arg \max _{k} b_{k}$, i.e., the maximum computing and bandwidth resource demands of all requests. Inequality (14) then can be rewritten as

$$
\frac{|\mathcal{R}(k)|}{|\mathcal{S}(k)|} \leq 8\left(C_{\max } \log \alpha+b_{\max } \log \beta\right) .
$$

The competitive ratio of algorithm Online_CP thus is

$$
\begin{aligned}
& \frac{|\mathcal{S}(k)|}{|O P T|} \geq \frac{|\mathcal{S}(k)|}{|\mathcal{R}(k) \cup \mathcal{S}(k)|} \geq \frac{|\mathcal{S}(k)|}{|\mathcal{R}(k)|+|\mathcal{S}(k)|}=\frac{1}{|\mathcal{R}(k)| /|\mathcal{S}(k)|+1} \\
& \geq \frac{1}{1+8\left(C_{\max } \log \alpha+b_{\max } \log \beta\right)}, \text { by inequality }(15) \\
& \geq \frac{1}{c^{\prime} \log |V|}, \text { when } C_{\max } \text { and } b_{\max } \text { are given constants, }
\end{aligned}
$$

where $c^{\prime}>0$ is a positive constant and $\alpha=\beta=2|V|$. The competitive ratio of the competition ratio of algorithm Online_CP thus is $O(\log |V|)$ when $\alpha=\beta=2|V|$.

The analysis of the time complexity of the proposed algorithm is omitted due to space limitation.

\section{PERFORMANCE EVALUATION}

In this section we evaluate the performance of the proposed algorithms through experimental simulation. We also investigate the impact of important parameters.

\section{A. Environment settings}

We consider SDNs consisting of from 50 to 250 nodes, where each network is generated using GT-ITM [6]. The number of servers in each network is set to $10 \%$ of the network size, and they are randomly co-located with switches in the network. We also use real network topologies, i.e., GÉANT [5] and an ISP network from [20]. There are nine servers for the GÉANT 
topology as set in [7] and the number of servers in the ISP networks are provided by [19]. The bandwidth capacity of each link varies from 1,000 Mbps to $10,000 \mathrm{Mbps}$ [11], and the computing capacity of each server varies from 4,000 to $12,000 \mathrm{MHz}$ [8]. Five types of network functions, i.e., Firewall, Proxy, NAT, IDS, and Load Balancing, are considered, and their computing demands are adopted from [7], [17]. The source and destination nodes of each multicast request is randomly generated, the ratio of the maximum number $D_{\max }$ of destinations of a multicast request to the network size $|V|$ is randomly drawn in the range of $[0.05,0.2]$, and its bandwidth resource demand is randomly drawn in the range of $[50,200] M b p s$. We set $\sigma_{e}$ and $\sigma_{v}$ at $|V|-1$. The maximum number $K$ of servers that can be used to implement the service chain of each multicast request is 3 . The running time of each algorithm is obtained based on a machine with a $3.40 \mathrm{GHz}$ Intel i7 Quad-core CPU and 16 GiB RAM. Unless otherwise specified, these parameters will be adopted in the default setting.

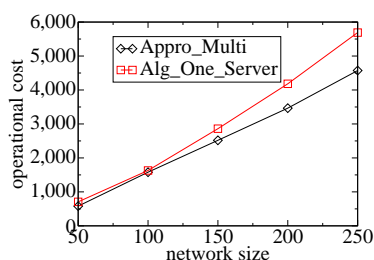

(a) Operational costs by different algorithms with $D_{\max } /|V|=$ 0.05

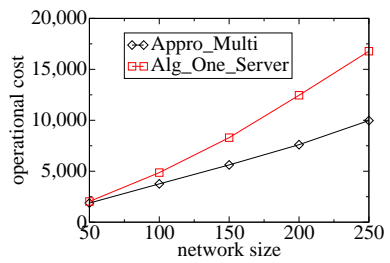

(c) Operational costs by different (d) Running times of different algorithms with $D_{\max } /|V|=$ algorithms with $D_{\max } /|V|=$ 0.2

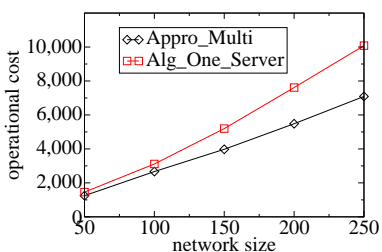

(b) Operational costs by different algorithms with $D_{\max } /|V|=$ 0.1
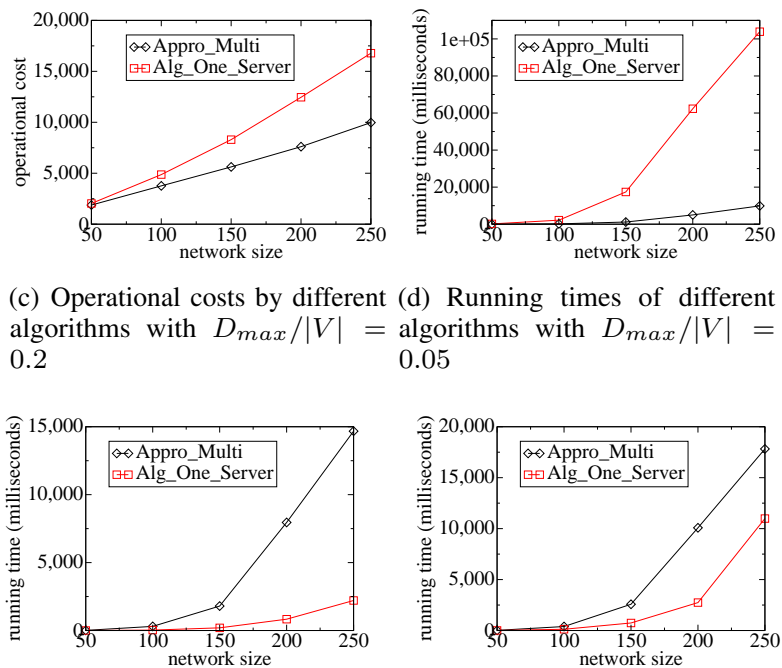

(e) Running times of different algorithms with $D_{\max } /|V|=0.1$

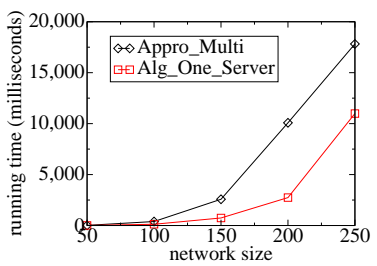

(f) Running times of different algorithms with $D_{\max } /|V|=0.2$

Fig. 5. The performance of algorithms Appro_Multi and Alg_one_Server

We evaluate the performance of algorithm Appro_Multi against the state-of-the-art - an algorithm in [22], referred to as algorithm Alg_one_Server, which only uses a single server to implement service chain $S C_{k}$ of each multicast request $r_{k}$. Namely, algorithm Alg_one_Server first routes the traffic of $r_{k}$ to a server, and then finds a Minimum Spanning Tree (MST) of a complete graph $G_{c}$ containing the destinations of $r_{k}$, where the edge between two destinations in $G_{c}$ represents the shortest path between the two nodes in the original network. It then expands the MST into its corresponding subgraph in the original network. It finally selects the combination of server and subgraph with the minimum cost.

For the online algorithms, we study the performance of algorithm Online_CP against those of a baseline heuristic SP. For multicast request $r_{k}$, algorithm SP first removes links and nodes that do not have enough available resources to admit $r_{k}$, and then assigns each link and each switch node in $V_{S}$ with the same weight. For each candidate server in $V_{S}$ it then finds a shortest path from $s_{k}$ to $v$ and a single-source shortest path tree rooted at $v$ and spanning all destinations of $r_{k}$. It finally uses a multicast tree with the minimum cost for $r_{k}$.

\section{B. Performance evaluation of approximation algorithms}

We first evaluate the performance of algorithm Appro_Multi against that of algorithm Alg_One_Server by varying the network size from 50 to 250 and the ratio of the maximum number $D_{\max }$ of destinations of each request to the network size $|V|$ from 0.05 to 0.2 . The operational cost and running time curves delivered by algorithms Appro_Multi and Alg_one_Server are drawn in Fig. 5, where the operational costs and running times are the average of admitting 1,000 NFV-enabled multicast requests. Specifically, we can see from Fig. 5 (a) that the operational cost by algorithm Appro_Multi is around 80\% of that of algorithm Alg_one_Server. The reason is that algorithm Appro_Multi may use multiple servers that are close to the destinations of the request to implement the service chain of the request, which can significantly reduce the cost of bandwidth resource usage. Furthermore, it can be seen from the figure that the performance gap between the two algorithms becomes larger and larger, with the increase on the network size. The rationale behind is that algorithm Appro_Multi has more chances to select a set of servers that are closer to the destinations of each request, considering that more servers in larger networks are to be chosen. The similar performance behaviour can be observed from Figs. 5(b) and (c). Furthermore, it can be seen from Figures 5 (d) - (f) that approximation algorithm Appro_Multi takes a slightly more time than that of algorithm Alg_One_Server, as different combinations of servers in $V_{S}$ are to be considered.

We then investigate the performance of approximation algorithms Appro_Multi and Alg_one_Server in real networks GÉANT and AS1755, by varying $\frac{\overline{D_{\max }}}{|V|}$ from 0.05 to 0.2 . Fig. 6 shows that the operational costs and running times of both algorithms. It can be seen that the operational cost delivered by algorithm Appro_Multi is much lower than that by algorithm Alg_one_Server while taking a slightly more running time. For example, the operational cost by algorithm Appro_Multi in network AS1755 is around 30\% lower than that of algorithm Alg_One_Server when $\frac{D_{\max }}{|V|}=0.15$ as shown in Fig. 6 (b).

The rest is to evaluate the performance of approximation algorithm Appro_Multi_Cap, by setting $\frac{D_{\max }}{|V|}$ at 0.2.Notice that algorithm Alg_one_Server is not considered as a benchmark as it does not consider SDN resource capacity 


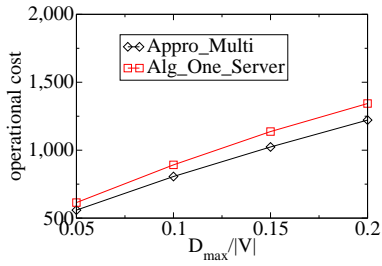

(a) Operational costs by different algorithms in network GÉANT

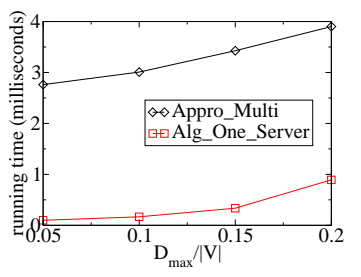

(c) Running times of different algorithms in network GÉANT

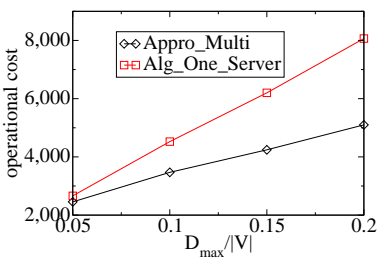

(b) Operational costs by different algorithms in network AS1755

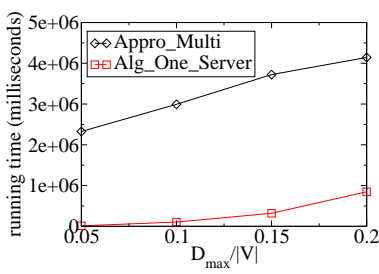

(d) Running times of different algorithms in network AS1755

Fig. 6. The performance of different algorithms in networks of GÉANT, AS1755, and AS4755

constraints. From Figs. 7(a) and 5(c), we can see that the operational cost of algorithm Appro_Multi_Cap is larger than that of algorithm Appro_Multi without capacity constraints. The reason is that the algorithm Appro_Multi_Cap excludes the servers and links without enough available resources from the consideration for an incoming multicast request, this may reduce the number of combinations of servers that can be explored to implement request.

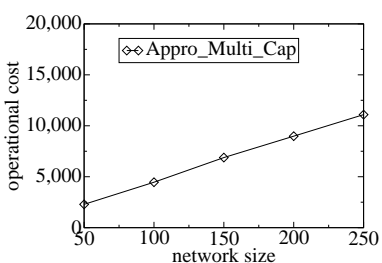

(a) The operational cost

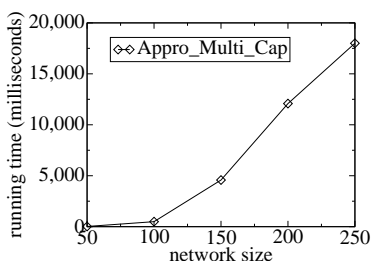

(b) The running time

Fig. 7. The performance of algorithms Appro_Multi_Cap

\section{Performance evaluation of online algorithms}

We then evaluate algorithm Online_CP against algorithm $\mathrm{SP}$, by varying the network size from 50 to 250 for a monitoring period that consists of 300 multicast requests. The numbers of admitted requests by algorithm Online_CP are shown in Fig. 8. Specifically, from Fig. 8 (a), we can see that online algorithm Online_CP admits at least twice the number of requests admitted by algorithm SP. Although the total resource capacity of each network keeps increasing with the increase on the network size, the number of requests admitted by each mentioned algorithm does not monotonically increase. This is because there is a higher probability that the destinations of each multicast request are far away from each other, thereby increasing the rejection probability of the request, due to more bandwidth resource consumptions.

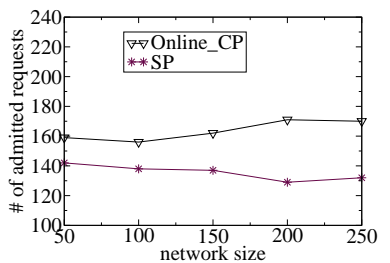

Fig. 8. The performance of online algorithms Online_CP and SP

We now evaluate the performance of algorithm Online_CP against that of online algorithm SP by varying the number of requests from 50 to 300, in GÉANT and AS1755, respectively. It can be seen from Fig. 9 (a) that algorithms Online_CP and SP can admit almost all requests if the number of requests is no greater 100. Otherwise, algorithm Online_CP admits more requests than that by algorithm SP. Also, the performance gap between them increases with the growth of the number of requests. The reason is that online algorithm Online_CP considers the workload (or the utilization) of each resource by assigning each resource an exponential cost, while algorithm SP does not take the resource workload and assigns the same weight to the same amount of resource at different nodes and links, which may lead to the excessive usage of a heavily-loaded resource. Similar performance of algorithms Online_CP and SP in network AS1755 can also be observed in Fig. 9 (b).

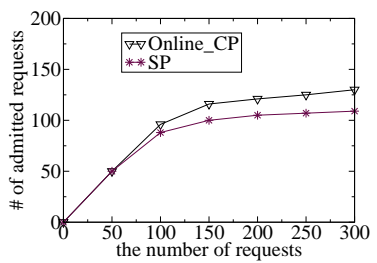

(a) Network GÉANT

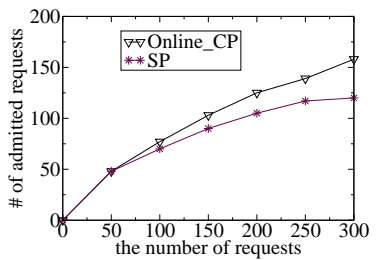

(b) Network AS1755
Fig. 9. The performance of algorithms Online_CP and SP

\section{CONCLUSION}

In this paper we studied NFV-enabled multicasting in an SDN. We first devised an approximation algorithm with a constant approximation ratio, assuming that the number of servers for implementing each service chain is no more than a constant $K \geq 1$, subject to the computing and bandwidth capacity constraints on servers and links in the network. We then studied dynamic admissions of NFV-enabled multicast requests without the knowledge of future request arrivals, with the objective to maximize the network throughput, for which we proposed an online algorithm with a competitive ratio if $K=1$. We finally evaluated the performance of the proposed algorithms by experimental simulations. Simulation results demonstrate that the proposed algorithms outperform the other heuristics.

\section{ACKNOWLEDGEMENTS}

The work by Zichuan $\mathrm{Xu}$ and Alex Galis is partially supported by the H2020 projects of SONATA (http://sonatanfv.eu) and 5GEx (https://5gex.tmit.bme.bu). 


\section{REFERENCES}

[1] Z. Cao, M. Kodialam, and T. V. Lakshman. Traffic steering in software defined networks: planning and online routing. Proc. of DCC, ACM, 2014.

[2] R. Cohen, L. Eytan, J. Naor, and D. Raz. On the effect of forwarding table size on SDN network utilization. Proc. of INFOCOM, IEEE, 2014.

[3] R. Cohen, L. Eytan, J. Naor, and D. Raz. Near optimal placement of virtual network functions. Proc. of INFOCOM, IEEE, 2015.

[4] M. R. Garey and D. S. Johnson. Computers and Intractability: A guide to the Theory of NP-Completeness. W.H. Freeman and Company, NY, 1979.

[5] GÉANT. http://www.geant. net.

[6] http://www.cc.gatech.edu/projects/gtitm/.

[7] A. Gushchin, A. Walid, and A. Tang. Scalable routing in SDN-enabled networks with consolidated middleboxes. Proc. of HotMiddlebox, ACM, 2015.

[8] Hewlett-Packard Development Company. L.P. Servers for enterprise bladeSystem, rack \& tower and hyperscale. ttp:// www8.p.com/us/en/products/servers/, 2015.

[9] L. Huang, H. Hung, C. Lin, and D. Yang. Scalable steiner tree for multicast communications in software-defined networking. Computing Research Repository (CoRR), vol. abs/1404.3454, 2014.

[10] M. Huang, W. Liang, Z. Xu, W. Xu, S. Guo and Y. Xu. Dynamic routing for network throughput maximization in software-defined networks. Proc. of INFOCOM, IEEE, 2016.

[11] S. Knight et al. The internet topology zoo. J. Selected Areas in Communications, Vol. 29, pp. 1765 - 1775, IEEE, 2011.

[12] L. Kou, G. Markowsy, and L. Berman. A faster algorithm for Steiner trees. Acta Informatica, Volume 15, pp. 141-145, Springer, 1981.

[13] T-W. Kuo, B-H. Liou, K. C. Lin, and M-J Tsai. Deploying chains of virtual network functions: on the relation between link and server usage. Proc. of INFOCOM, IEEE, 2016.

[14] Y. Li, L. T. X. Phan, and B. T. Loo. Network functions virtualization with soft real-time guarantees. Proc. of INFOCOM, IEEE, 2016.

[15] T. Lukovszki and S. Schmid. Online admission control and embedding of service chains. Proc. of SIROCCO, 2015.

[16] L. Mamatas, S. Clayman, and A. Galis. Software-defined infrastructure. IEEE Communications Magazine, Vol. 53, No. 4, pp 166-174, IEEE, 2015.

[17] J. Martins et al. ClickOS and the art of network function virtualization. Proc. of NSDI, USENIX, 2014.

[18] H. Moens and F. D. Turck. VNF-P: A model for efficient placement of virtualized network functions. Proc. of CNSM, IEEE, 2014.

[19] Z. A. Qazi, C. C. Tu, L. Chiang, R. Miao, V. Sekar, M. Yu. SIMPLEfying middlebox policy enforcement using SDN. Proc. of SIGCOMM, ACM, 2013.

[20] N. Spring, R. Mahajan, and D. Wetherall. Measuring ISP topologies with rocketfuel. Proc. of SIGCOMM, ACM, 2002.

[21] Y. Zhang et. al. StEERING: A software-defined networking for inline service chaining. Proc. of ICNP, IEEE, 2013.

[22] S. Q. Zhang, Q. Zhang, H. Bannazadeh, and A. L. Garcia. Network function virtualization enabled multicast routing on SDN. Proc. of ICC, IEEE, 2015.

[23] S. Q. Zhang, Q. Zhang, H. Bannazadeh, and A. L. Garcia. Routing algorithms for network function virtualization enabled multicast topology on SDN. IEEE Transaction on Network and Service Management, Vol.12, No.4, pp.580-594, 2015. 